\title{
Prediction of the electrical characteristics of heterostructural microwave devices with transverse current transfer based on a quantum-mechanical self-consistent model of a nanoscale channel with taking into account inter-valley scattering
}

\author{
Natalia Vetrova ${ }^{1, *}$, Yury Ivanov $^{1}$, Evgeny Kuimov $^{1}$, Mstislav Makeev ${ }^{1}$, Sergey Meshkov ${ }^{1}$, \\ Kirill Pchelintsev ${ }^{1}$, and Vasily Shashurin ${ }^{1}$ \\ ${ }^{1}$ Department of Radioelectronics and Laser Technologies, Bauman Moscow State Technical \\ University (National Technological University), Moscow 105005, Russia Federation
}

\begin{abstract}
A mathematical model of current transfer in AlGaAsheterostructures with taking into account inter-valley dispersion and space charge in the process of degradation is presented. The developed computational algorithm is optimized by the criterion of temporal and spatial complexity. The relative deviation of the calculation results from the experimental data on the curvature of the initial portion of the currentvoltage characteristic of the resonant tunnel diodes on AlGaAsheterostructures is less than $3 \%$.
\end{abstract}

\section{Introduction}

Nanoelectronics devices based on semiconductor $\mathrm{A}_{3} \mathrm{~B}_{5}$ nanoscale heterostructures are of interest as the basis for ultrafast devices of the new generation [1,2]. Research results [3,4] show that such devices have the potential for applications in industries with high reliability requirements. Aluminum-gallium arsenide (AlGaAs) is the most common material for heterostructures of the $A_{3} B_{5}$ compounds. Silicon doping is usually used to control the conductivity of the contact layers in such structures.

The task of predicting the current-voltage characteristics (CVC) of AlGaAsheterostructures arises both in the design of microwave devices based on them, and in evaluation of their reliability. To increase the accuracy of the calculation in the model of current transfer, the inclusion of such processes as inter-valley dispersion and Coulomb interaction of electrons is included. However, the algorithms of such models are characterized by high temporal complexity and need to be refined to analyze structures subject to degradation changes.

* Corresponding author: natasha_vetrova@mail.ru 


\section{Simulation}

To take into account the inter-valley scattering, a multichannel model was used, within which each channel was assigned a valley in the dispersion diagram. Since the transitions between the $\Gamma$ - and X-valleys are most energetically favorable for a pair of GaAs/AlAs, the transitions between other valleys are not take $n$ into account. The wave functions of electrons in the $\Gamma$ - and X-channels were calculated using the Schrödinger equation for an open system [5]. The definition of the operator of communication between channels, presented in [6], was generalized to the case of a continuous aluminum profile in order to be able to model structures subject to degradation.

$$
A=\alpha\left|\frac{d x}{d z}\right|
$$

where $\alpha$ - constant of inter-valley interaction, $x$ - aluminum mole fraction, $z$ - coordinate.

The Schrödinger equation was solved by the finite difference method. The optimization of the spatial complexity of the computational algorithm was carried out on the basis of a five-diagonal matrix representation using the theory of sparse matrices and the coordinate method of their storage. When taking into account the electron-electron interaction, the introduction of a self-consistent potential into the Hamiltonian [7, 8] is the most timeconsuming part of the calculation of the electron concentration. In order to reduce the time complexity of the algorithm, modified formulas for calculating the concentration were developed.

\section{Results}

The figures below present the results of calculating the wave functions of electrons in the single-valley and two-valley approximation for the two-barrier structure. As can be seen from Fig. 1, when calculating in the two-valley approximation, new metastable levels appear in the $\Gamma$-valley, corresponding to metastable levels in the X-channel, through which electrons can tunnel through the channel.

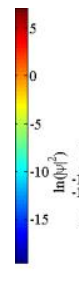

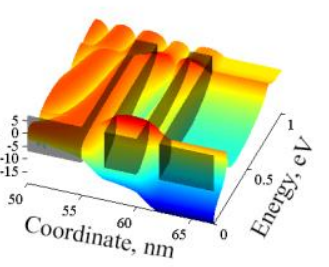

a)
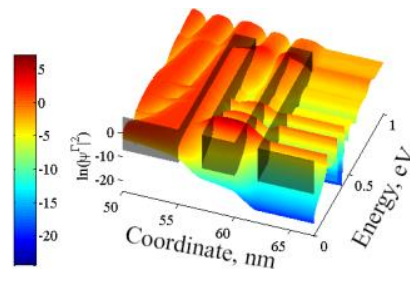

b)

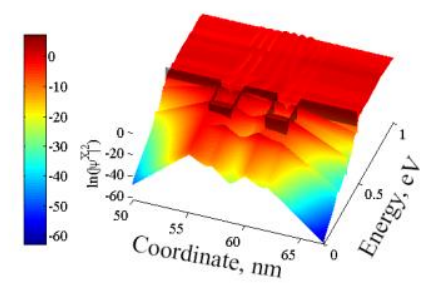

c)

Fig. 1. The electron density probabilities calculated in the framework of the one-valley (a) and two-valley approximations $(\mathrm{b}-\Gamma$-valley, $\mathrm{c}-\mathrm{X}$-valley).

The presented models were used to calculate the current-voltage characteristics of a number of resonant-tunneling diodes, the results are presented in Fig. 3. The diffusion profile changes at the fabrication stage were taken into account. As can be seen from the graphs, a satisfactory agreement between the calculation results and the experiment has been achieved. It was found that the best results are achieved when the constant of intervalley interaction is equal to $0.7 \mathrm{eV} \cdot \AA$. Calculated according to formula 2 , the relative 
deviation of the calculation results from the experiment according to the criterion of curvature of the initial part of the CVC is $3 \%$.

$$
\Delta C=\int_{0}^{1}\left(J n_{e}-J n_{p}\right)^{2} d x\left(\int_{0}^{1} J n_{e}^{2} d x\right)^{-1}
$$

where $\Delta C$ - relative deviation $J n_{f}$ and $J n_{p}$ - experimental and calculated normalized CVC, which is calculated as $J n(x)=J_{p}^{-1} J\left(x V_{p}\right)$, where $V_{p}$ - peak voltage, $J_{p}$ - peak current density
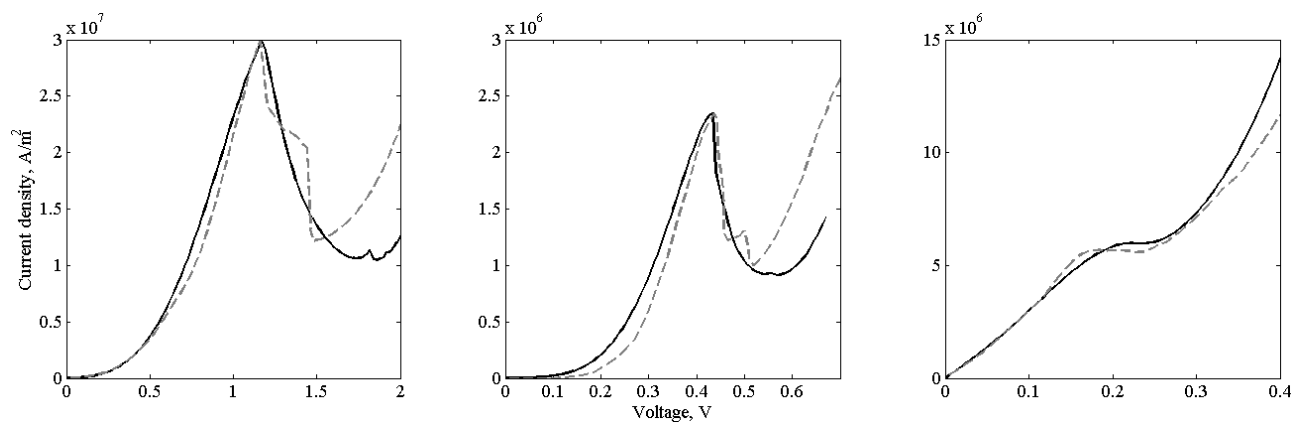

Fig. 2. The calculation results (black line) in comparison with the experiment (grey dotted line).

\section{Conclusion}

A mathematical model of transverse current transfer in heterostructural microwave devices subject to degradation processes at elevated temperatures during the production phase, as well as during operation, with taking into account a inter-valley scattering and an influence of the space charge is presented. The developed computational algorithm is optimized by the criterion of temporal and spatial complexity including by increasing the efficiency of the self-consistency algorithm at the stage of calculating the concentration. Comparison of simulation results with experiment suggests that it is advisable to use the developed algorithms to predict electrical characteristics as well as reliability indicators of heterostructural microwave devices with transverse current transfer, the assignment parameters of which are determined by the initial part of the $\mathrm{CVC}$ of the structures.

\section{References}

1. S. Nadar, M. Zaknoune, X. Wallart, High performance heterostructure low barrier diodes for sub-THz detection, IEEE Transactions on Terahertz Science and Technology, v. 7(6), pp. $780-788$ (2017)

2. K. C. Magruder, A.F.J. Levi, Optimal design of heterostructure tunnel diode with nonlinear current-voltage characteristic, Physica E, v. 44, pp. 1503-1509 (2012)

3. Y. Sin, N. Presser, Z. Lingley, M. Brodie, B. Foran, S. Moss, Reliability, Failure Modes, and Degradation Mechanisms in High Power Singleand Multi-Mode InGaAsAlGaAs Strained Quantum Well Lasers, Proc. of SPIE, v. 9733(973304) (2016)

4. D. Bonsendorf, S. Schneider, J. Meinschien, J. W. Tomm, Reliability of high power laser diodes with external optical feedback, Proc. of SPIE, v. 9733(973302) (2016)

5. S. Datta. Quantum Transport. Atom to Transistor (Cambridge University Press, Cambridge, 2005)

6. I.I. Abramov, I.A. Goncharenko, N.V. Kolomejtseva, Two-band combined model of resonant tunneling diode, Physics of the solid state, v. 41(11), pp.1395-1400 (2007) 
7. J. Wang, E. Wasige, Self-consistent analysis of the IV characteristics of resonant tunnelling diodes, International Journal of Terahertz Science and Technology, v. 5(4), pp. 153-162 (2012)

8. J.F. Mennemann, H. Kosina, A. Jungel, Transient Schrodinger-Poisson Simulations of a High-Frequency Resonant Tunneling Diode Oscillator, Journal of Computational Physics, v. 239, pp. 187-205 (2013) 